Research Article

\title{
Impact of co-blocking the costimulatory signals on immune-related genes after high-risk rabbit corneal allograft using 2nd-generation DNA sequencing technology
}

\author{
Hai-Xia Zhao ${ }^{1}$ iD , Xin-Yu Li ${ }^{1}$ iD, Wen-Ying Guan ${ }^{1}$ (iD), Xiao-Tong $\operatorname{Han}^{1}$ iD \\ ${ }^{1}$ Center of Myopia, The Affiliated Hospital of Inner Mongolia Medical University, Hohhot, China.
}

\begin{abstract}
The aim of this study was to evaluate the impact and mechanism of co-blocking of costimulatory signals CD28-B7CD40-CD40L during immune allograft rejection. Forty-eight recipient rabbits were prepared as a high-risk corneal allograft model. After surgery, the animals were randomly divided into: control group, MR1 group, anti-B7 group, and co-blocking group ( $n=12$, each group). Subconjunctival injection was first performed on the allograft surgery day until post-surgery day five. Four weeks later, or when immune rejection occurred, the cornea was sampled to detect and analyze the gene spectrum. The survival time in the co-blocking group was significantly longer than that in the other three groups $(p<0.05)$. Gene expression analysis revealed that the expression of genes associated with immune rejection, interleukin (IL)-1 $\alpha, \mathrm{IL}-1 \beta$, intercellular cell adhesion molecule-1, and IL-2 was down-regulated in the co-blocking group, while IL-10 was up-regulated, but the changes in nuclear factor-kB and interferon- $\gamma$ were not significant. In conclusion, the co-blocking of costimulatory signals can significantly reduce genes that promote corneal allograft rejection. The inhibition of corneal allograft rejection gene expression was significantly enhanced. These gene expression results can explain the conclusion of previous work at the genetic level.
\end{abstract}

Keywords: Costimulatory signal, corneal allograft, immune rejection, second-generation sequencing technology, antibody.

Received: May 31, 2018; Accepted: October 15, 2018

\section{Introduction}

According to statistics, the blindness of $2-3$ million patients is caused by corneal disease (Cornea Group of Ophthalmology Society, Chinese Medical Association, 2014), and this disease has become the second leading cause of blindness (Gong et al., 2015). The only cure for corneal disease is corneal allograft (Zhang et al., 2015). Due to the corneal structure, the graft is relatively immune-tolerant and the rejection rate is low, with success rate higher than that of other organs (Niederkorn, 2013; Yin et al., 2014). However, recent studies have shown that although the incidence of postoperative rejection is low, it can still reach 10-20\% (Paunicka et al., 2015; Blanco and Saban, 2015; Choi et al., 2015). Furthermore, high risk patients such as those with neovessels, persistent inflammation, and secondary allograft, the incidence of immune rejection may reach 50\% (Tan et al., 2012). Therefore, preventing the occurrence of rejection after high-risk corneal allograft and improving post-keratoplasty graft survival have become key concerns for many ophthalmologists (Dohlman et al., 2015).

Send correspondence to Hai-Xia Zhao. Center of Myopia, The Affiliated Hospital of Inner Mongolia Medical University, No. 1 of Tongdao North Street, the Huis District, Hohhot 010050, China. E-mail: zhaohx521463@163.com
In the present study, a high-risk rabbit corneal allograft model was established, in which two antibodies were used to co-block CD28-B7 and CD40-CD40L in the costimulatory pathway (Kirk et al., 1997; Ye et al., 2014). Then gene expression changes in the corneal graft were investigated to evaluate the impact of co-blocking CD28-B7 and $\mathrm{CD} 40-\mathrm{CD} 40 \mathrm{~L}$ in the costimulatory pathway on allograft rejection at the gene level. The goal of the present study was to compare graft survival time and to analyze gene expression of different stimulation pathways on high-risk rabbit corneal allograft rejection.

\section{Material and Methods}

\section{Animals}

Seventy-two healthy adult New Zealand white rabbits were provided by the Laboratory Animal Center of Inner Mongolia Medical University. Of the total, 48 rabbits were recipients and 24 were donors. Rabbits weighed within 2-3 $\mathrm{kg}$, without male/female limit. The present study was carried out in strict accordance with the recommendations in the Guide for the Care and Use of Laboratory Animals of the National Institutes of Health. The animal use protocol was reviewed and approved by the Institutional Animal Care and Use Committee (IACUC) of Inner Mongolia 
Medical University. All rabbits were raised in a laboratory, and the shed met the criterion for experimental rabbit general environmental requirements, such as room temperature within $16-26^{\circ} \mathrm{C}$, day temperature differences of $\leq 4{ }^{\circ} \mathrm{C}$, relative humidity of $40-70 \%$, minimum air changes of 8 $\mathrm{h} / \mathrm{time}$, noise of $\leq 60 \mathrm{db}$ (A), illumination of 100-200 lx, and an alternation of day and night time of 12/12 hours or 10/14 hours. There was no special requirement on feeding and drinking for the experimental animals.

\section{Establishment of the high-risk corneal allograft model}

The suture method was used to induce neovascularization. First, all animals were injected with $20 \%$ urethane solution (3-4 mL/kg) (Sun et al., 2016) into the ear vein for anesthesia. Then, $0.4 \%$ oxybuprocaine (Sun et al., 2016) hydrochloride eye drops were used for surface anesthesia in the right eyes of rabbits. Lastly, four sutures were uniformly stitched in the partial central corneal lamina area with $0-5$ suture, and the stitch length was approximately 5 $\mathrm{mm}$. Approximately two weeks after the neovessels formed, rabbits with neovessels larger than three quadrants were selected as high-risk recipients for corneal allograft. Before performing penetrating keratoplasty, $1 \%$ pilocarpine (Zhao et al., 2013) nitrate eye drops were used to narrow the pupils of the right eyes, three times a day, and $0.2 \%$ gentamicin (Sun et al., 2016) was used to rinse the conjunctival sac of right eyes. Then, all animals were injected with $20 \%$ urethane solution $(3-4 \mathrm{~mL} / \mathrm{kg})$ into the ear vein for anesthesia, and the rabbits were allowed to lie on the left side. Then, $0.4 \%$ oxybuprocaine hydrochloride eye drops were used for surface anesthesia via the right eye of the rabbits. Lastly, routine aseptic operation was conducted after disinfection. Under an operating microscope, one $7.25-\mathrm{mm}$ trephine (diameter) was used to sample the graft from the corneal surface, and one 7.0-mm trephine (diameter) was used to prepare the cornea for the recipient planting bed. A 10-0 nylon suture was used to apply 16 interrupted sutures. After surgery, $800 \mathrm{U}$ of gentamicin was injected (Sun et al., 2016), and $0.5 \mathrm{~mL}$ of $0.5 \%$ dexamethasone (Sun et al., 2016) mixture was applied into the subconjunctiva of the right eyes. In addition, $1 \%$ atropine eye ointment and tobramycin dexamethasone eye ointment $(0.3 \%$ tobramycin and $0.1 \%$ dexamethasone) were also applied (Sun et al., 2016) to the right eye, which was covered with a sterile eye pad. At the second day after surgery, tobramycin dexamethasone eye drops $(0.3 \%$ tobramycin and $0.1 \%$ dexamethasone) were applied three times a day, $0.5 \%$ compound of tropicamide eye drops were applied once a day, and tobramycin dexamethasone eye ointment $(0.3 \%$ tobramycin and $0.1 \%$ dexamethasone) was applied once a day.

\section{Animal grouping and processing}

After surgery, the animals were randomly divided into four groups $(\mathrm{n}=12)$. Subconjunctival injection was ini- tially performed on the allograft surgery day, and was continued until post-surgery day five. The control group was injected with saline, the animals in the MR1 group were injected with anti-CD40L monoclonal antibody (Abnova, Taipei, Taiwan) at $100 \mu \mathrm{g} / \mathrm{day}$, the animals in the anti-B7 group were injected with anti-B7 antibody (Abnova) at 100 $\mu \mathrm{g} / \mathrm{day}$, and the animals in the co-blocking group were injected with MR1 antibody and anti-B7 antibody at 100 $\mu \mathrm{g} /$ day (each).

\section{Postoperative observation}

After surgery, neovessels, graft edema, and turbidity were observed under a slit lamp microscope to assess growth of the planting bed. The allograft rejection index (RI, 0-12 points) was recorded, and these three scores were added to determine the RI on the observation day. The scoring method was as follows: Corneal neovascularization (CNV): no CNV, 0 point; one quadrant of $\mathrm{CNV}, 1$ point; two quadrants of $\mathrm{CNV}, 2$ points; three quadrants of $\mathrm{CNV}, 3$ points; four quadrants of $\mathrm{CNV}, 4$ points. Corneal edema: no corneal edema, 0 point; mild edema, 1 point; mild-tomoderate edema, 2 points; subepithelial micro-vesicle, 3 points; bullous keratopathy, 4 points. Corneal turbidity: transparent corneal, 0 point; mild turbidity, 2 points; corneal aggravated turbidity (anterior chamber structure could be identified with two points): obvious turbidity, and anterior chamber structure is vague, three points; corneal has white turbidity, and anterior chamber cannot be observed, four points. When $\mathrm{RI} \geq 6$, cornea rejection occurred, which was considered the graft survival time.

\section{Sampling and specimen preparation}

When rejection occurred at four weeks after the allograft, the rabbits were sacrificed using the air embolism method, and the cornea was rapidly separated and stored at $-80^{\circ} \mathrm{C}$. After extracting and purifying the RNA (Trizol method), sequencing was performed using a Solexa sequencer (Illumina, San Diego, CA, USA), according to the manufacturer's instructions, and the data were compared and analyzed. The corneal tissue RNA results of the control and experimental groups are presented in Table 1 . The results were 2100 RIN $\geq 7.0$ and $28 \mathrm{~S} / 18 \mathrm{~S} \geq 0.7$ of the four samples, which show that the samples are qualified, and the quality of the RNA was in good condition.

\section{Statistical analysis}

SPSS13.0 statistical software (SPSS Inc., Chicago, IL, USA) and GraphPad Prism 5.0 (GraphPad Software Inc., San Diego, CA, USA) were used for data analysis. Data are reported as (mean \pm SD). Significance was determined with the Log rank $t$-test for comparing post-allograft graft survival time of the four groups; $p<0.05$ was considered statistically significant. Clustering analysis, GO func- 
Table 1 - Corneal tissue RNA extraction results.

\begin{tabular}{|c|c|c|c|c|c|c|c|}
\hline Number & Sample group & $\begin{array}{c}\text { Concentration } \\
(\mu \mathrm{g} / \mu \mathrm{L})\end{array}$ & $\begin{array}{l}\text { Quantity before } \\
\text { purification }(\mu \mathrm{g})\end{array}$ & $\mathrm{A} 260 / \mathrm{A} 280$ & 2100RIN & $28 \mathrm{~S} / 18 \mathrm{~S}$ & Result \\
\hline A & MR1 group & 0.1163 & 11.0 & 2.13 & 9.4 & 1.7 & qualified \\
\hline B & Anti-B7 group & 0.8998 & 85.5 & 2.06 & 7.0 & 1.4 & qualified \\
\hline G & Drug combination group & 0.0830 & 7.9 & 2.12 & 9.7 & 2.0 & qualified \\
\hline $\mathrm{C}$ & Control group & 0.1288 & 12.2 & 2.02 & 7.1 & 1.2 & qualified \\
\hline
\end{tabular}

Annotation: qualified: $2100 \mathrm{RIN} \geq 7.0$ and $28 \mathrm{~S} / 18 \mathrm{~S} \geq 0.7$

tional significance, and pathway enrichment analysis were carried out on the differentially expressed genes.

\section{Results}

\section{Comparison of graft survival time and survival rate}

The post-allograft graft survival time of the four groups had a normal distribution and the variances were homogeneous. As shown in Figure 1, the survival times of animals in the four groups were the same $(p<0.0001)$. Pairwise comparison revealed that grafts in the co-blocking group had a longer mean survival time of $55.75 \pm 7.51$ days, which was significantly different compared to the other groups $(p<0.05)$. Furthermore, the control group exhibited a significantly shorter survival time, when compared to the

\section{Survival proportions}

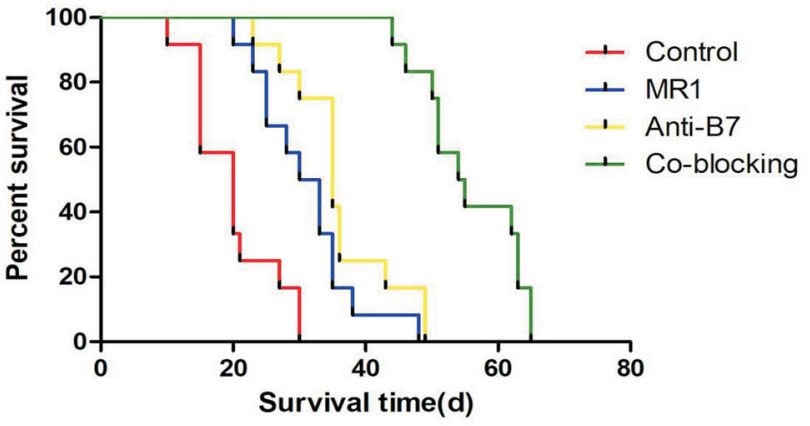

Figure 1 - Survival curve of transplanted corneal graft in different groups. (A) survival curve of rabbits in the control group; (B) survival curve of rabbits in the MR1 group; (C) survival curve of rabbits in the Anti-B7 group; (D) survival curve of rabbits in the co-blocking group. Compared with the control group, the co-blocking group displayed a significantly longer allograft survival time. Abscissa: survival time; Ordinate: percent of survival. other groups $(19.83 \pm 6.68$ days, $p<0.05)$. The median post-allograft graft survival time of control group, MR1 group, Anti-B7 group, and co-blocking group were 20, $31.5,35$ and 54.5 days, respectively.

\section{Gene sequencing analysis}

The pretreatment results of sequencing data quality are shown in Table 2. The inspection of the base distribution was used to detect the presence of the AT and GC separation phenomenon, which may have been caused by the sequencing or building the library, thereby affecting the subsequent quantitative analysis. In addition, theoretically, $\mathrm{G}$ and $\mathrm{C}$ bases, as well as $\mathrm{A}$ and $\mathrm{T}$ bases, on each sequence cycle should be equal and kept stable throughout the whole process. For RNA-seq, there were large fluctuations on the sequencing of few of the bases before each read, due to a random primers amplification bias, which is normal. The results revealed that sample stability and detection rate completely met the requirement.

A total of 1,463 genes were found differentially expressed between the MR1 group and control group, among which 741 genes were up-regulated and 722 down-regulated. Furthermore, 1,367 genes were differentially expressed between the anti-B7 group and control group, among which 618 genes were up-regulated and 749 downregulated. A total of 1,325 genes were differentially expressed between the co-blocking group and control group, among which 273 genes were up-regulated and 1,052 down-regulated (Figures 2-4). A total of 1,527 genes were differentially expressed between the co-blocking group and MR1 group, among which 230 genes were up-regulated and 1,297 down-regulated. In addition, 1,739 genes were differentially expressed between the co-blocking group and anti-B7 group, among which 166 genes were up-regulated

Table 2 - Pretreatment results of sequencing data quality.

\begin{tabular}{lcccccc}
\hline Sample & Raw reads & Raw bases & Clean bases & Valid ratio (base) & Q30 (\%) & GC content (\%) \\
\hline MR1 group & 34000000 & 4250000000 & 4214703900 & $99.16 \%$ & $90.01 \%$ & $55.00 \%$ \\
Anti-B7group & 31869184 & 3983648000 & 3950967418 & $99.17 \%$ & $90.35 \%$ & $54.00 \%$ \\
Drug combination group & 34000000 & 4250000000 & 4216166011 & $99.20 \%$ & $90.83 \%$ & $53.00 \%$ \\
Control group & 30478448 & 3809806000 & 3777116010 & $99.14 \%$ & $89.37 \%$ & $53.00 \%$ \\
\hline
\end{tabular}

Q30: percentage of bases with Phred values greater than 30 of total base number.

General requirements are of less than $85 \%$. 


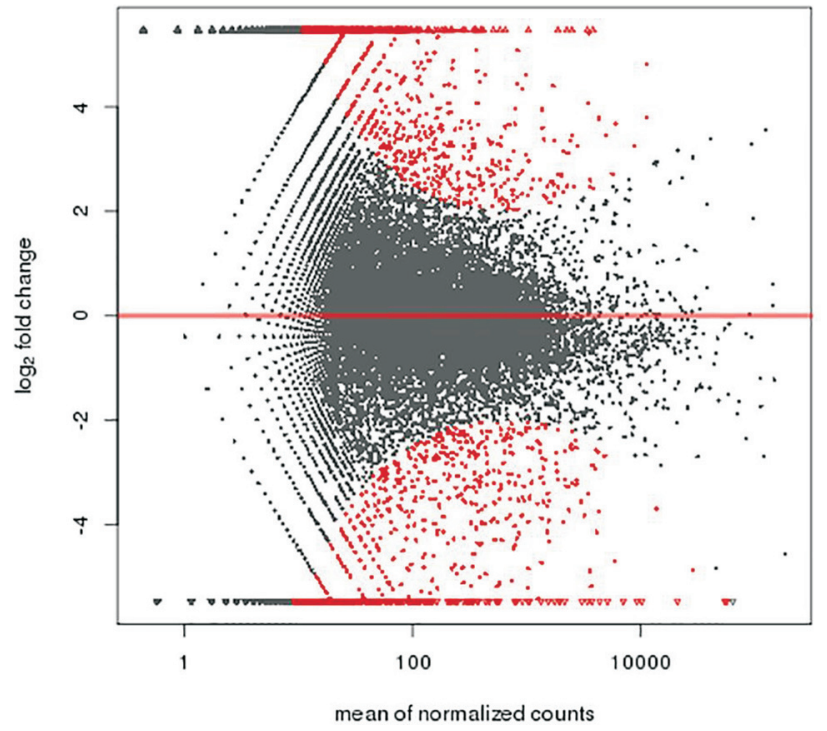

Figure 2 - A total of 1,463 genes were differentially expressed between the MR1 group and control group, among which 741 genes were found up-regulated and 722 genes were down-regulated. Abscissa: mean of normalized counts; Ordinate: $\log _{2}$ fold change.

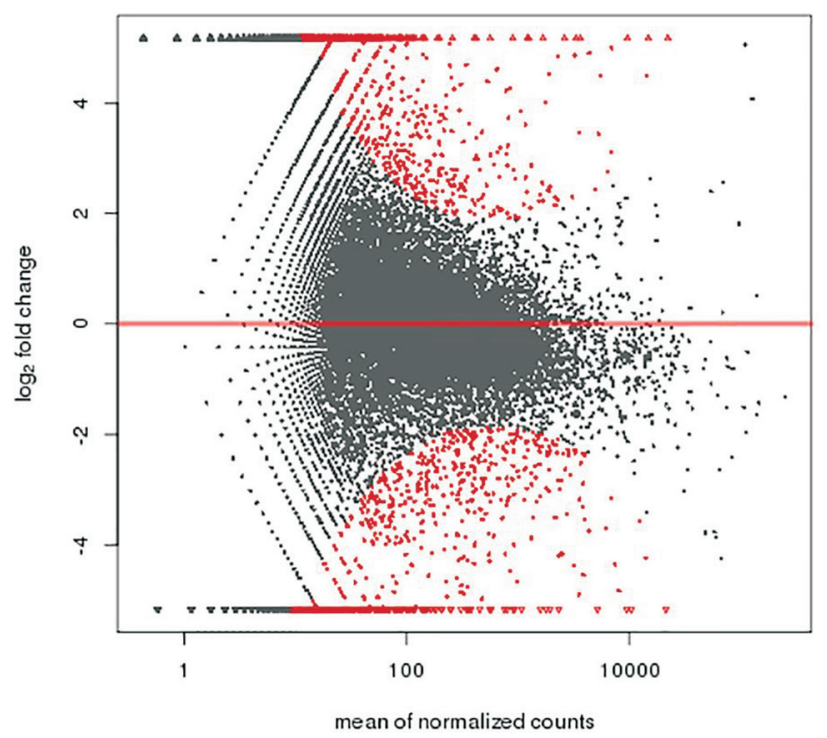

Figure 3 - A total of 1,367 genes were differentially expressed between the anti-B7 group and control group, among which 618 genes were up-regulated and 749 down-regulated. Abscissa: mean of normalized counts; Ordinate: $\log _{2}$ fold change.

and 1,573 down-regulated (Figures 5 and 6). The default screening difference was set as $p \leq 0.05$.

\section{Differentially expressed genes}

Table 3 lists the gene expression changes in the MR1 group and anti-B7 group. The expression of interleukin (IL)-10 was up-regulated, the expression of intercellular cell adhesion molecule-1 (ICAM1) did not change, and the expression of the others was downr-egulated. Between the

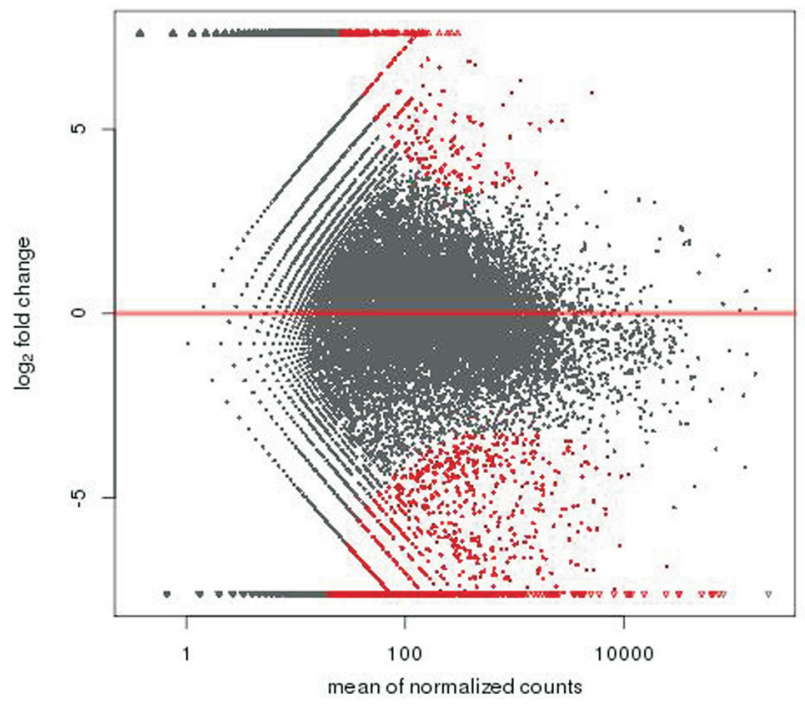

Figure 4 - A total of 1,325 genes were differentially expressed between the co-blocking group and control group, among which 273 genes were up-regulated and 1,052 down-regulated. Abscissa: mean of normalized counts; Ordinate: $\log _{2}$ fold change.

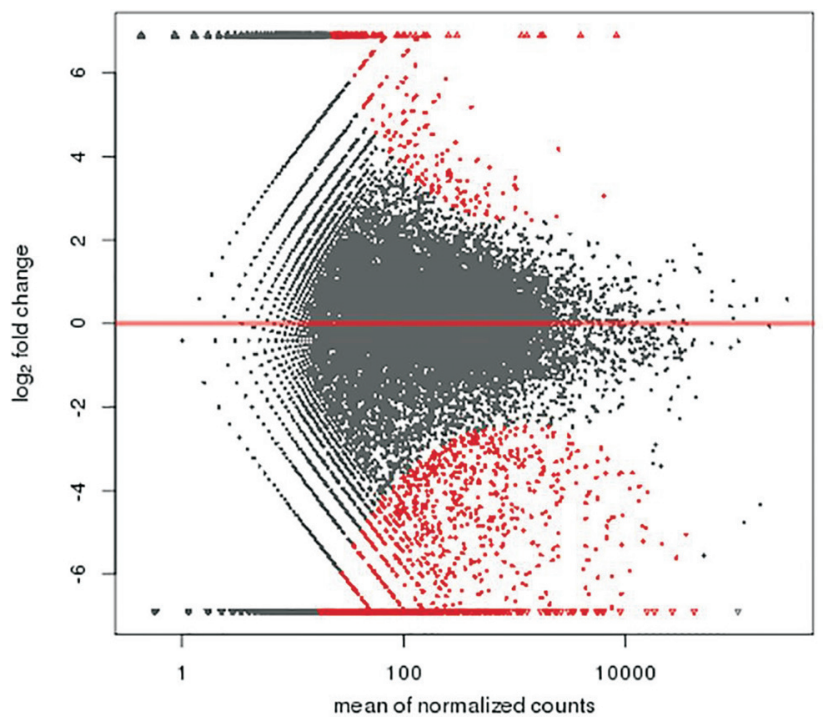

Figure 5 - A total of 1,527 genes were differentially expressed between the co-blocking group and MR1 group, among which 230 genes were up-regulated and 1,297 down-regulated. Abscissa: mean of normalized counts; Ordinate: $\log _{2}$ fold change.

co-blocking group and the other three groups, nuclear factor (NF)-kB, IL-1 $\beta$, IL-1 $\alpha$ and IL-2 were down-regulated, the expression of IL-10 was up-regulated, and the expression of interferon- $\gamma($ IFN- $\gamma)$ did not change.

This experiment required a fold-change value of $\geq 2$ for a gene to be considered differentially expressed. As shown in Table 4, IFN- $\gamma$ was differentially expressed only in the MR1 group, NF- $\kappa \mathrm{B}$ was not differentially expressed, and ICAM-1 was differentially expressed only in the co- 


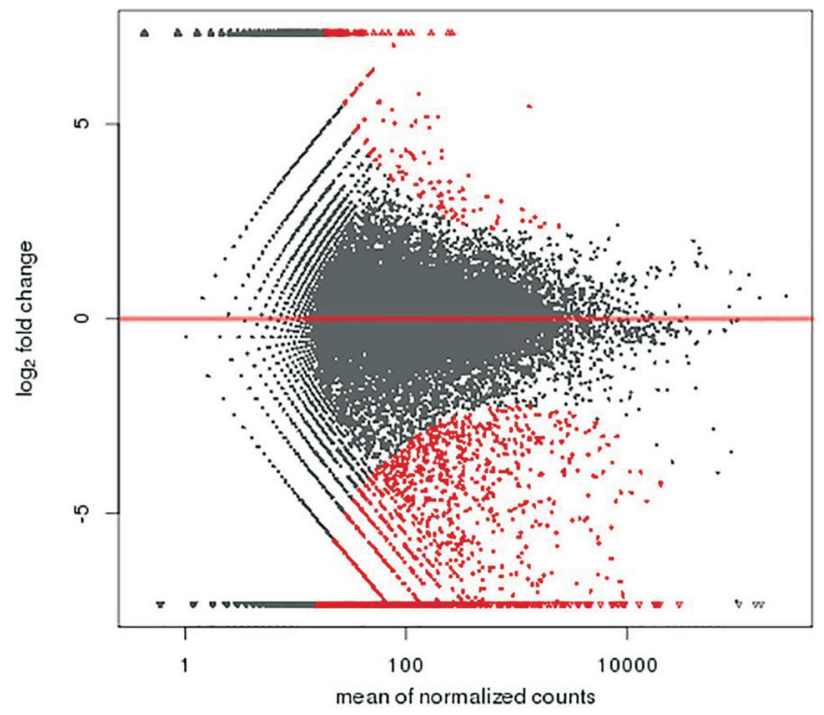

Figure 6 - A total of 1,739 genes were differentially expressed between the co-blocking group and anti-B7 group, among which 166 genes were up-regulated and 1,573 downr-egulated. Abscissa: mean of normalized counts; Ordinate: $\log _{2}$ fold change.

blocking group. Furthermore, IL-1, IL-2, and IL-10 were differentially expressed. Compared with the other monotherapy groups, the IL-1, IL-2, and IL-10 genes exhibited significantly different expression.

\section{Discussion}

An increasing number of studies have revealed costimulatory signaling pathways in corneal allograft rejection, such as CD28-B7, CD40-CD40L, and B7-ICOS (Ye et al., 2014). Among these, the CD28 and CD40 signaling pathways are important in the field of clinical allograft, since they complement each other. Previous studies of organ allografts revealed that co-blocking of CD28-B7 and CD40-CD40L had a synergistic effect. Kirk et al. (1997) inhibited these two pathways in a kidney allograft model in rhesus monkeys, and found that the co-blocking effects were better than those obtained when blocking one pathway alone, and the survival period was extended to more than 150 days. In addition, these two pathways were independent. Simply blocking the CD40 or CD28 pathway reduced corneal allograft rejection.

CD40 and CD28 are transmembrane glycoproteins, while $\mathrm{CD} 40 \mathrm{~L}$ and $\mathrm{B} 7$ are their primary ligands, respectively. The binding of the ligand and receptor may initiate the costimulatory signal, thereby activating $\mathrm{T}$ cells to proliferate and differentiate into effector cells and produce effects (Kolly et al., 2007). Howland et al. (2000) verified that the response of $\mathrm{T}$ cells to antigens in the absence of CD28 and CD40. CD28 plays an important role in initiating $\mathrm{T}$ cell responses, whereas CD40 maintains the reactions. The inhibition of CD28 prevents the differentiation of $\mathrm{Th} 2$ cells, and inhibiting CD40 may be more efficient for the

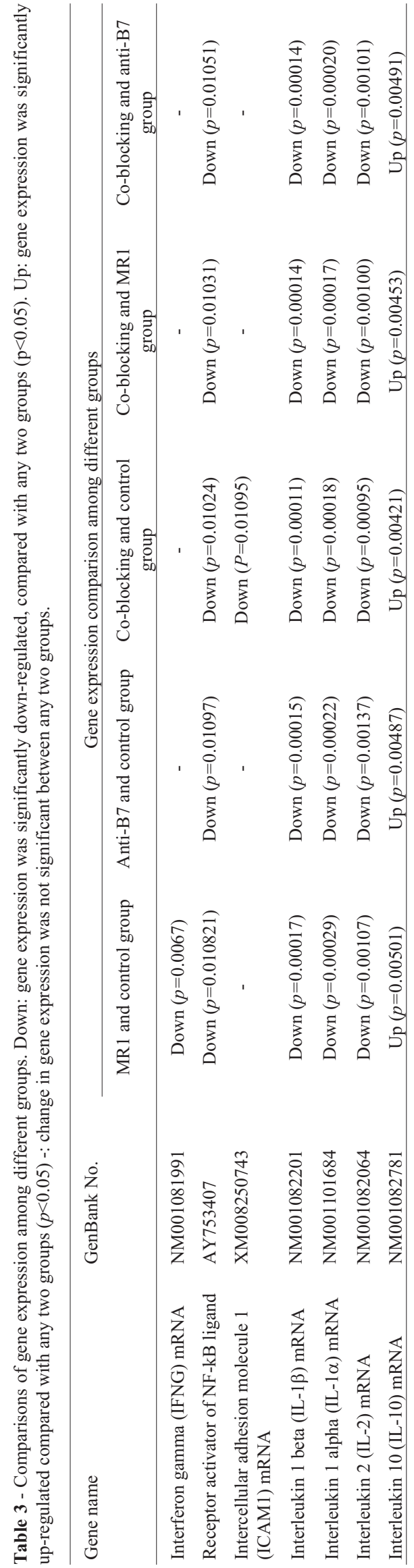




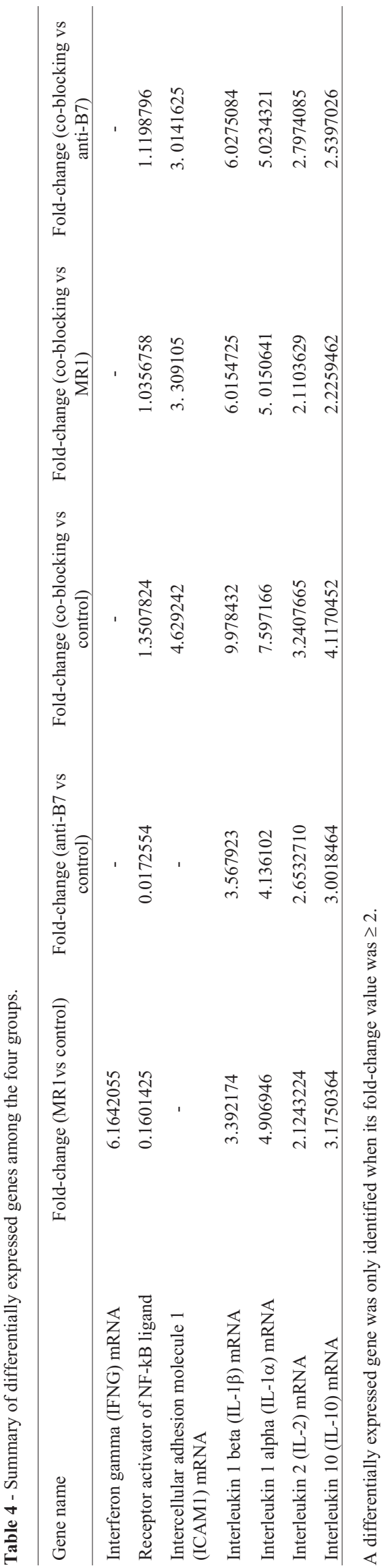

production of Th1 cytokines. Therefore, blocking the CD28 pathway may inhibit $\mathrm{T}$ cell activation, while blocking the CD40 pathway may inhibit Th1 cell differentiation and maintain $\mathrm{T}$ cell reactions.

In the present study, the corresponding antibodies were injected under the conjunctiva of the rabbit high-risk corneal allograft model, and these competitively bound the ligand to inhibit the aggregation of the signaling complex and terminate the activation of $\mathrm{T}$ cells. Thus, the activation and maintenance of the immune response were inhibited. The ultimate goal was to avoid T cell-mediated immune rejection.

Our experiments confirmed that co-blocking CD28B7 and CD40-CD40L costimulatory signals significantly inhibited high-risk corneal allograft rejection, and graft survival time was longer than in the single block mode. Furthermore, the present study compared corneal tissues in the control group, MR1 group, anti-B7 group, and co-blocking group at the gene level, as we performed RNA sequencing to screen for differentially expressed genes. Thus, the impact of co-blocking CD28-B7 or CD40-CD40L costimulatory signals on high-risk corneal rejection was examined. This experiment focused on immune rejection-related genes, such as IFN- $\gamma$, NF- $\kappa$ B, ICAM- 1 , IL- $1 \alpha$, IL- $1 \beta$, IL-2, and IL-10.

IFN- $\gamma$ is mainly produced by activated Th1 cells. It functions as an effective monocyte/macrophage inducer to induce the expression of major histocompatibility complex-II-class antigens on the cell surface, and strengthen antigen presentation and specific immune recognition processes. King et al. (2000) found that rats without cornea surgery or corneal graft rejection revealed no IFN- $\gamma$ mRNA expression in the corneal graft, and that the IFN- $\gamma$ mRNA level in the graft significantly increased by 9-13 days after surgery when allograft rejection occurred, suggesting that IFN- $\gamma$ plays a role in corneal graft rejection. Skurkovich et al. (2002) used an anti-IFN- $\gamma$ antibody in human penetrating keratoplasty. They found that visual improvement and graft opacity were better than before surgery, and no change was observed after three months of surgery, indicating that the anti-IFN- $\gamma$ antibody significantly suppressed corneal graft rejection. In the present study, the IFN- $\gamma$ gene revealed no significant change in expression, probably because when immunity occurred or the corneal graft was removed 28 days later, the IFN- $\gamma$ mRNA level had already decreased, and could not be detected at the gene level.

$\mathrm{NF}-\mathrm{\kappa B}$ is a nuclear transcription factor with broad roles and present in nearly all cells in humans. Under normal conditions, NF- $\mathrm{KB}$ exists in a non-active form in the cytoplasm. When cells are stimulated by specific factors, such as IL-1 or tumor necrosis factor- $\alpha$, the inhibitory protein is released and NF- $\kappa B$ enters the nucleus. NF- $\mathrm{KB}$ can regulate the expression of specific genes involved in inflammation and the immune response, such as cytokines 
(IL-2, IL-12, vascular endothelial growth factor, and tumor necrosis factor- $\alpha$ ), adhesion molecules (ICAM-1 and VCAM-1), and chemokines (IL-8, MCP-1 and RANTES), which affect immune responses (Afonina et al., 2015; Proto et al., 2015). The proliferation and differentiation of $\mathrm{T}$ cells, B cells, lymphocytes, and dendritic cells also requires the participation of $\mathrm{NF}-\kappa \mathrm{B}$, and it plays an important role in humoral and cellular immunity.

Cell adhesion is a condition of inflammation, immunity, and neovascularization, and ICAM-1 is an important cell adhesion molecule. ICAM-1 can induce leukocytes to adhere to activated vascular endothelial cells, thereby promoting leukocytes to migrate towards the graft and mediate interactions between $\mathrm{T}$ cells and the corresponding target cells. In addition, ICAM-1 can stimulate antigen-presenting cells, thereby activating $\mathrm{T}$ lymphocytes to differentiate into effector T cells. ICAM-1 can also help transfer the activation signals of Langerhans cells to T cells. After gathering different leukocyte subsets towards the inflammation sites, ICAM-1 can initiate or enhance inflammatory and immune reactions (Demmers et al., 2015). Thus, ICAM-1 is closely correlated to corneal graft rejection and can effectively inhibit the synthesis and secretion of ICAM-1, which has become an important objective in anti-rejection treatment.

IL-1 is a cytokine that functions in the early stage of infections and is extremely important for promoting corneal allograft rejection. IL-1 is expressed in most nucleated cells, as well as in the normal cornea. There are two types of IL-1, IL- $1 \alpha$ and IL- $1 \beta$ that have similar functions and can bind to the same receptors. IL- $1 \beta$ is the dominant form. BenEzra et al. (1990) reported that IL-1 strongly influences the formation of new blood vessels, and copy number variation affects the occurrence of corneal rejection. Under antigen stimulation, IL-1 levels are significantly increased to regulate the acute inflammatory phase, produce chemotaxis, and activate inflammatory cells and antigen-presenting cells, thereby activating $\mathrm{T}$ cells and $\mathrm{B}$ cells. In addition, collagenase levels and adhesion molecule expression are increased.

According to the gene expression results, IL-1 $\alpha$, IL$1 \beta$, ICAM-1, and IL-2 are down-regulated in the coblocking group compared to the monotherapy group. These genes promote corneal allograft rejection. Thus, along with the weakened expression of these genes, rejection was effectively suppressed. IL-10 expression was found upregulated in the co-blocking group when compared to the monotherapy groups, causing inhibitory effects on corneal allograft rejection. Thus, along with the enhanced expression of IL-10, rejection was significantly reduced, and graft survival time in the co-blocking group was significantly longer than that in the other three groups. NF- $\kappa \mathrm{B}$ and IFN- $\gamma$ did not exhibit any significant changes in expression level, which should be further evaluated by selecting different time points after removing the corneal allograft.

The following limitations of the present study must be acknowledged. First, due to the small sample size and low number of selected genes, further comparisons and analyses need to be conducted in studies with a larger sample size and additional genes. Second, because of the differences between rabbit and human eyes, the test remains a controversial method to assess the ophthalmic drug action in humans. Finally, although the co-blocking of CD28-B7 and CD40-CD40L costimulatory signals can prolong corneal allograft survival time, the anti-rejection effects of co-blocking of CD28-B7 and CD40-CD40L over longer periods require further study. Despite these limitations, this is one of the first studies to reveal the impact and mechanism of co-blocking of CD28-B7 and CD40-CD40L costimulatory signals on corneal allograft rejection.

In conclusion, the results of the present study revealed that the co-blocking of CD28-B7 and CD40-CD40L costimulatory signals can significantly reduce the expression of genes that promote corneal allograft rejection. At the same time, the inhibition of corneal allograft rejection gene expression was significantly enhanced.

\section{Acknowledgments}

This study was supported by the Science and Technology Projects of Inner Mongolia Autonomous Region, 2015 (kjt15sf13), the Natural Science Foundation of Inner Mongolia Autonomous Region, China, 2013 (2013MS1185), and thee Natural Science Foundation of Inner Mongolia Autonomous Region in 2017 (2017MS(LH)0830).

\section{Conflict of Interest}

The authors have expressed no conflict of interest regarding this paper.

\section{Author Contributions}

HXZ, conceived and designed the study, HXZ, XYL, WYG and XTH conducted the experiments, HXZ, XYL, WYG and XTH analyzed the data, HXZ wrote the manuscript, XYL, WYG and XTH revised it critically for important intellectual content; all authors read and approved the final version.

\section{References}

Afonina IS, Elton L, Carpentier I and Beyaert R (2015) MALT1 a universal soldier: Multiple strategies to ensure NF- $\kappa$ B activation and target gene expression. FEBS J 282:3286-3297.

BenEzra D, Hemo I and Maftzir G (1990) In vivo antigenic activity of interleukins. Arch Ophthalmol 108:573-576.

Blanco T and Saban DR (2015) The cornea has the nerve to encourage immune rejection. Am J Transplant 15:1453-1454. 
Choi HJ, Lee JJ, Kim DH, Kim MK, Lee HJ, Ko AY, Kang HJ, Park C and Wee WR (2015) Blockade of CD40-CD154 costimulatory pathway promotes long-term survival of fullthickness porcine corneal grafts in nonhuman primates: Clinically applicable xenocorneal allograft. Am J Transplant 15:628-641.

Cornea Group of Ophthalmology Society, Chinese Medical Association (2014) The most progression of corneal and ocular surface disease research in China. Zhonghua Yan Ke Za Zhi 50:695-698.

Demmers MW, Korevaar SS, Roemeling-van Rhijn M, van den Bosch TP, Hoogduijn MJ, Betjes MG, Weimar W, Baan CC and Rowshani AT (2015) Human renal tubular epithelial cells suppress alloreactive $\mathrm{T}$ cell proliferation. Clin Exp Immunol 179:509-519.

Dohlman TH, Omoto M, Hua J, Stevenson W, Lee SM, Chauhan SK and Dana R (2015) VEGF-trap aflibercept significantly improves long-term graft survival in high-risk corneal allograft. Transplantation 99:678-686.

Gong YB, Hu LN, Liu Y, Han GC, Guo HL, Luo L, Wang LQ, Li Y and Huang YF (2015) Effects of foxp3 gene modified dendritic cells on mouse corneal allograft rejection. Int $\mathbf{J}$ Clin Exp Med 8:3965-3973.

Howland KC, Ausubel LJ, London CA and Abbas AK (2000) The role of CD28 and CD40 ligand in T cell action and tolerance. J Immunol 164:4465-4470.

King WJ, Comer RM, Hudde T, Larkin DF and George AJ (2000) Cytokine and chemokine expression kinetics after corneal allograft. Transplantation 70:1225-1233.

Kirk AD, Harlan DM, Armstrong NN, Davis TA, Dong Y, Gray GS, Hong X, Thomas D, Fechner Jr JH and Knechtle SJ (1997) CTLA4-Ig and anti-CD40 ligand prevent renal allograft rejection in primate. Proc Natl Acad Sci U S A 94:8789-8794.

Kolly R, Thiel MA, Herrmann T and Plückthun A (2007) Monovalent antibody $\mathrm{scFv}$ fragments selected to modulate T-cell activation by inhibition of CD86-CD28 interaction. Protein Eng Des Sel 20:91-98.
Niederkorn JY (2013) Corneal allograft and immune privilege. Int Rev Immunol 32:57-67.

Paunicka KJ, Mellon J, Robertson D, Petroll M, Brown JR and Niederkorn JY (2015) Severing corneal nerves in one eye induces sympathetic loss of immune privilege and promotes rejection of future corneal allografts placed in either eye. Am J Transplant 15:1490-1501.

Proto JD, Tang Y, Lu A, Chen WC, Stahl E, Poddar M, Beckman SA, Robbins PD, Nidernhofer LJ, Imbrogno K et al. (2015) NF- $\kappa B$ inhibition reveals a novel role for HGF during skeletal muscle repair. Cell Death Dis 6:e1730.

Skurkovich S, Kasparov A, Narbut N and Skurkovich B (2002) Treatment of corneal transplant rejection in humans with anti-interferon-gamma antibodies. Am J Ophthalmol 133:829-830.

Sun L, Zhao H and Guan WY (2016) Effects of combined block of co-stimulating signalon rabbit anti-rejection after neovascularization corneal transplantation. Rec Adv Ophthalmol 36:809-812.

Tan DT, Dart JK, Holland EJ and Kinoshita S (2012) Corneal allograft. Lancet 379:1749-1761.

Ye QX, Xu LH, Xu W and Fang JP (2014) Influence of blocking B7/CD28 and CD40/CD154 co-stimulatory signals on immune function of sensitized mice. Zhongguo Shi Yan Xue Ye Xue Za Zhi 22:801-806.

Yin XT, Tajfirouz DA and Stuart PM (2014) Murine corneal allograft: A model to study the most common form of solid organ allograft. J Vis Exp 93:e51830.

Zhang H, Wang L and Zhang L (2015) Cyclosporine nanomicelle eye drop: A novel medication for corneal graft allograft treatment. Biol Pharm Bull 38:893-900.

Zhao H, Luo Y, Niu C and Guan W (2013) Comparison of treatments for bullous keratopathy in rabbits. Exp Ther Med 5:1481-1485.

Associate Editor: Jorge Lopez-Camelo

License information: This is an open-access article distributed under the terms of the Creative Commons Attribution License (type CC-BY), which permits unrestricted use, distribution and reproduction in any medium, provided the original article is properly cited. 